\title{
Evaluation of the Completeness in the Filling of Laboratory Request Forms Submitted to the Haematology Laboratory at a Tertiary Hospital in Kenya
}

\author{
Japheth C. Kipkulei*, Teresa C. Lotodo \\ Department of Haematology and Blood Transfusion, School of Medicine, College of Health Sciences, Moi University, Eldoret, \\ Kenya \\ Email: ^jkipkulei@gmail.com
}

How to cite this paper: Kipkulei, J.C. and Lotodo, T.C. (2019) Evaluation of the Completeness in the Filling of Laboratory Request Forms Submitted to the Haematology Laboratory at a Tertiary Hospital in Kenya. Health, 11, 862-868.

https://doi.org/10.4236/health.2019.117069

Received: April 6, 2019

Accepted: July 8, 2019

Published: July 11, 2019

Copyright $\odot 2019$ by author(s) and Scientific Research Publishing Inc. This work is licensed under the Creative Commons Attribution International License (CC BY 4.0).

http://creativecommons.org/licenses/by/4.0/

(c) (i) Open Access

\begin{abstract}
Background information: The laboratory request form is a communication link between the clinicians and the laboratory staff. It contains demographic details of the patient including full names, age and gender; the test required; location of the patient; date and time of request among other details. Incomplete and inaccurate filling of the request forms may cause errors which can impact on the quality of the patient care. Methodology: The study was a descriptive cross sectional where 289 laboratory request forms submitted consecutively to the haematology laboratory during the month of January 2018 were evaluated. Data was collected using a data collection form and analyzed by use of frequency table. Ethical clearance was sought from Institutional Research and Ethical Committee of Moi University and Moi Teaching and Referral Hospital. Results: Of all the 289 forms evaluated, only 1\% (3/289) had all the required information. The parameters with the most information were patient's name (100\%), hospital number (100\%), sex $(99.7 \%)$, age $(98.3 \%)$, investigation requested (97.6\%), the location of the patient $(96.9 \%)$, clinician's name (96.9\%) and signature (96.5\%). The least information was recorded for clinical history (14.7\%) and address of the patient (4.8\%). Conclusion: The study showed that there were inadequacies in the recording of the required information in the laboratory request form. The parameters which were completely filled were the patient's name and the hospital number, whereas the address and the clinical history had the least completion rates.
\end{abstract}

\section{Keywords}

Laboratory, Request Forms, Required Information, Completion 


\section{Introduction}

The laboratory plays an important role in patient care. It is an essential component of quality health care delivery. The laboratory request form is a communication link between the clinicians and the laboratory staff. Studies have shown that $60 \%-70 \%$ of clinical decisions about diagnoses, prescription, diagnosis and monitoring of treatment are based on laboratory data [1].

George Lundberg introduced the concept of brain-to-brain loop for laboratory diagnosis over 30 years ago [2]. According to Lundberg's model, the first step in the loop involves the selection of an appropriate laboratory test(s) in the brain of the physician caring the patient which is then communicated through the laboratory request form. This is followed by numerous intermediary steps, such as identification of the patient, specimen collection and specimen handling; and then by the actual specimen analysis in the laboratory. The last steps involve the release of test results for the physician's review and reaction to the laboratory information, the interpretation of the results and the implementation of appropriate clinical action.

Traditionally laboratory testing is divided into three phases, pre-analytical, analytical and post-analytical. Evaluation of the laboratory request form is an example of auditing the pre-analytical phase of the laboratory process [3]. The information required on a laboratory request form includes the demographic data of the patient such as name(s), date of birth, address, age, and sex. Other details include the patient's location and destination of report, hospital number, diagnosis, the investigation(s) requested, type of specimen, name and signature of the clinician requesting the test, telephone number of the doctor; clinical details including relevant medication and the date and time of request.

All the three phases of laboratory testing may be subjected to errors and studies have shown that errors in laboratory can occur in all the phases, though it is well established that most errors occur in the pre-analytical and post-analytical phases [4]. Pre-analytical errors can occur at the time of patient assessment, test order entry, request completion, patient identification, specimen collection, specimen transport, or specimen receipt in the laboratory. Bonini et al. [5] found that pre-analytical errors predominated and accounted for $31.6 \%$ to $67 \%$. A study in Kenya [6] looking at errors during the three phases of laboratory testing found that preanalytical errors were most common with a frequency of 148 (42.8\%), followed by analytical errors 114 (32.9\%) and post analytical errors 84 $(24.3 \%)$. In this study request forms lacking the address accounted for $27.0 \%$ of the pre-analytical errors.

Medical errors are known to impact negatively on patient outcome and increase the cost of health care [7]. Incomplete filling of the laboratory request forms may lead to delay in reporting of the laboratory results and this would delay starting of treatment, especially of critically ill patients. Moreover, lack of information on the laboratory request form may impact on the interpretation of the results by the requesting clinician and hence may lead to wrong diagnosis. 
Every attempt therefore, to reduce laboratory errors, including accurate and complete filling of the laboratory request form by the clinicians has to be undertaken to ensure quality and efficient patient care.

\section{Methodology}

This was a descriptive cross-sectional study carried out at Moi Teaching and Referral Hospital which is a 900-bed capacity hospital located in Eldoret town, Kenya and it serves western Kenya, parts of Eastern Uganda and South Sudan.

The laboratory request forms submitted to the haematology laboratory in the month of January 2018 were evaluated. A total of 289 laboratory request forms were consecutively sampled during the study period. The sample size was calculated using the following formula:

$$
n=z^{2} p q / d^{2}
$$

where:

$n=$ the desired sample size.

$z=$ the corresponding value confidence level of $95 \%$ in the normal distribution table which is 1.96 .

$P=$ the proportion of laboratory request forms which are completely filled (17.8\%) [8].

$q=1-p$ (Proportion of the LRF which are incompletely filled (82.2\%).

$d=$ the degree of precision, which was set at $0.05 \%$.

$n=1.96^{2} \times 0.178 \times 0.822 / 0.05^{2}=225$.

Data was collected using a data collection form, entered into SPSS software version 20 and analyzed using frequency tables. Ethical approval was obtained from Institutional Research and Ethical Committee (IREC) of Moi University and Moi Teaching and Referral Hospital. The patients' personal information was de-identified.

\section{Results}

A total of two hundred and eighty-nine request forms were audited. Only 3/289 $(1.0 \%)$ of the request forms contained all the required information and the patient's name and the hospital number were present on all the forms evaluated. The patient's age and sex were documented on $98.3 \%$ and $99.7 \%$ of the forms respectively, whereas the address and the occupation of the patients were missing on $95.2 \%$ and $95.8 \%$ of forms respectively. Only 43 (14.9\%) of the request forms evaluated contained the clinical history of the patient.

With respect to physician information; the name of the clinician ordering the test was provided on $96.9 \%$ of the forms, while $96.5 \%$ were signed by the clinician. The type of specimen, the date and time the test was ordered and the investigation requested were present on $84.4 \%, 95.5 \%, 84.1 \%$ and $97.6 \%$ of the 289 forms respectively. The information required on laboratory request forms and the proportion of laboratory forms that contained the required information is shown in Table 1. 
Table 1. Proportion of the laboratory request forms with the required information.

\begin{tabular}{lcc}
\hline \multicolumn{1}{c}{ Information required } & Present (n) & $\%$ \\
\hline Name of patient & 289 & 100 \\
Age & 284 & 98.3 \\
Sex & 288 & 99.7 \\
Hospital number & 289 & 100 \\
Address & 14 & 4.8 \\
Occupation & 12 & 4.2 \\
Type of specimen & 244 & 84.4 \\
Date of specimen collection & 276 & 95.5 \\
Time of collection & 243 & 84.1 \\
Investigation requested & 282 & 97.6 \\
Diagnosis & 248 & 85.8 \\
Clinical history & 43 & 14.9 \\
Location of the patient & 280 & 96.9 \\
Name of clinician & 280 & 96.9 \\
Signature of clinician & 279 & 96.5 \\
\hline
\end{tabular}

\section{Discussion}

There were only $1.0 \%(3 / 289)$ of the evaluated request forms with all the required information and the rest had one or more of the parameters missing. This result is similar to the finding by Oyedeji et al. [9] and Oyelekan et al. [10] of $1.3 \%$ and $0.2 \%$ respectively. On the patient identifiers, the name and the hospital number were recorded in all the laboratory request forms and this compares to that reported by Makubi et al. [8], Olayemi \& Asiamah-Broni [11]. and Jegede et al. [12]. The hospital number is important in identification of patients because the patients may share same or similar first names and surnames.

The age and the sex of the patients were present in $98.3 \%$ and $99.7 \%$ of the forms respectively and this finding concurs with the results by Jegede et al. [12]. and Makubi et al. [8]. Patient's demographic data is crucial as they enable the laboratory physician to interpret certain tests appropriately, for example the reference intervals in complete blood count are age and sex specific. Also, some diseases are more common in certain age groups.

The date of specimen collection was recorded in $95.5 \%$ of the laboratory request forms. Jegede et al. [12] reported a similar finding. The time of collection of the specimen was indicated in $84.1 \%$ of the forms and this was higher than the findings reported in studies by Adekoge et al. [13], Makubi et al. [8], Oyelekan et al. [10] and Olayemi \& Asiamah-Broni [11] where the parameter was recorded in $10.3 \%, 1.5 \%, 0.7 \%$ and $0.0 \%$ of the request forms respectively. The date and time of specimen collection are important because delay in delivery of some specimens may impact on the test results, e.g. delay in delivery blood specimen can lead to storage changes of the blood cells and this could complicate interpretation of test results [14] [15]. This information is also necessary when 
turn-around time is being considered or complaints about delays in reporting arise.

In terms of clinical information, the diagnosis was filled in $85.8 \%$ of the request forms and this finding compares with the that by Nutt et al. [16] of $80.9 \%$ but higher than the result by Makubi et al. [8] of 37.9\%. The clinical history was indicated in $14.9 \%$ of the forms and it is lower than the one reported by Makubi et al. [8] of $73.8 \%$ and Olayemi \& Asiamah-Broni [11] of 77.3\%. Provision of adequate clinical information has been demonstrated to help in correct interpretation of test results and in prevention of inappropriate investigations (Burton \& Stephenson, 2001 [17] [18]. Absence of clinical information or misleading information leads also to extraneous and unnecessary additional tests and thus unwarranted costs. In addition, inadequate clinical information may lead to misleading and potentially harmful comments made on laboratory results [1].

The location (ward/clinic) of the patient was provided in $96.9 \%$ of the request forms. This was comparable to results from studies by Nutt et al. [16], Jegede et al. [12] and Adekoge et al. [13] of $95.1 \%, 100 \%$ and $99.7 \%$ respectively but contrasts with that by Makubi et al. [8] and Olayemi \& Asiamah-Broni [11] of $86.75 \%$ and $52.2 \%$ respectively. Information on the location is important in that it helps in locating the patient and or the attending clinician in order to deliver test results, especially in cases of test results (critical values) which require urgent action by the attending clinician.

Clinician's name and sign were available in $96.9 \%$ and $96.5 \%$ of the evaluated forms respectively and this finding is similar to that from studies by Oyelekan et al. [10] and Jegede et al. [12] where both the parameters were present in $93.8 \%$ and $90.1 \%$ respectively. However, our finding differs from the results from studies by Makubi et al. [8] and Olayemi \& Asiamah-Broni [11] where the information was present in $88.7 \%$ \& $7.7 \%$ and $55.5 \%$ \& $55.2 \%$ of the laboratory request forms respectively. Our laboratory request forms, due to its design did not have a place for recording the contact address or telephone number of requesting clinician and also the details (name and contacts) of the attending clinician. The contact details of the clinicians are required in cases where a follow up is needed or critical test results are to be communicated.

One of the limitations of this study is that we did not seek the opinion of the healthcare workers involved in completing the laboratory request forms. This would have given more insight into the reasons for the incomplete forms. In addition, the laboratory requests were not classified in terms of the rank of the ordering clinician (medical intern, medical officer, registrar or consultant) which could have enlightened on the possible relationship between the cadre of the ordering clinician and the quality of completion of the request forms.

\section{Conclusion}

The study showed that there were inadequacies in the recording of the required information in the laboratory request form. The parameters which were com- 
pletely filled in the request forms were the patient's name and the hospital number, whereas the address and the clinical history had the least completion rates. This inadequacy may impact negatively on the interpretation of results, timely communication of critical values, turnaround times and ultimately the quality of patient care. A study to determine the reasons for the inadequate filling of the laboratory request forms by the clinicians should be carried out. However, there is need to increase the awareness of clinicians by way of continuous medical education on the importance of adequate and complete filling of laboratory request forms in patient care. The significance of each parameter on the request form should be emphasized. In addition, the hospital should redesign the laboratory request form to include the telephone number of the patient and the ordering clinician. The contact details of the attending clinician should also be included in the new request form. Furthermore, specimens being accompanied by inadequate and or incomplete laboratory request forms should be rejected by the laboratory staff.

\section{Conflicts of Interest}

The authors declare no conflicts of interest regarding the publication of this paper.

\section{References}

[1] Plebani, M. (2006) Errors in Clinical Laboratories or Errors in Laboratory Medicine? Clinical Chemistry and Laboratory Medicine, 44, 750-759. https://doi.org/10.1515/CCLM.2006.123

[2] Plebani, M., Favaloro, E.J. and Lippi, G. (2012). Patient Safety and Quality in Laboratory and Hemostasis Testing: A Renewed Loop? Seminars in Thrombosis and Hemostasis, 38, 553-558. https://doi.org/10.1055/s-0032-1315960

[3] Erasmus, R.T. and Zemlin, A.E. (2009) Clinical Audit in the Laboratory. Journal of Clinical Pathology, 62, 593-597. https://doi.org/10.1136/jcp.2008.056929

[4] Plebani, M. (2010) The Detection and Prevention of Errors in Laboratory Medicine. Annals of Clinical Biochemistry, 47, 101-110. https://doi.org/10.1258/acb.2009.009222

[5] Bonini, P., Plebani, M., Ceriotti, F. and Rubboli, F. (2002) Errors in Laboratory Medicine. Clinical Chemistry, 48, 691-698.

[6] Kimengech, K.K., Waithaka, S.K., Onyuka, J. and Kigondu, C.S. (2016) Determination of Errors that Compromise the Quality of Laboratory Service in a Tertiary Hospital. Asian Journal of Medical Sciences, 8, 64-70. https://doi.org/10.3126/ajms.v8i1.14740

[7] Institute of Medicine (US) Committee on Quality of Health Care in America. (2000) To Err is Human: Building a Safer Health System. In: Kohn, L.T., Corrigan, J.M. and Donaldson, M.S., Eds., National Academies Press, Washington DC. https://www.ncbi.nlm.nih.gov/books/NBK225182/

[8] Makubi, A.N., Meda, C., Magesa, A., Minja, P., Mlalasi, J., Salum, Z., et al. (2012) Audit of Clinical-Laboratory Practices in Haematology and Blood Transfusion at Muhimbili National Hospital in Tanzania. Tanzania Journal of Health Research, 14. https://ora.ox.ac.uk/objects/uuid:2b3c136a-a2c7-4ff7-a94f-c368c738a35c 
[9] Oyedeji, O.A., Ogbenna, A.A. and Iwuala, S.O. (2015) An Audit of Request Forms Submitted in a Multidisciplinary Diagnostic Center in Lagos. The Pan African Medical Journal, 20, 423. https://doi.org/10.11604/pamj.2015.20.423.5778

[10] Oyelekan, A.A., Ojo, O.T., Olawale, O.O., Adeleye, O.O., Sogebi, O.A., Osinupebi, O.A. and Olatunji, P.O. (2018) Pattern of Completion of Laboratory Request Forms in a Tertiary Health Facility. Annals of Health Research, 4, 155-161.

[11] Olayemi, E. and Asiamah-Broni, R. (2011) Evaluation of Request Forms Submitted to the Haematology Laboratory in a Ghanaian Tertiary Hospital. The Pan African Medical Journal, 8, 33. http://www.ncbi.nlm.nih.gov/pmc/articles/PMC3201597/

[12] Jegede, F., Mbah, H.A., Dakata, A., Gwarzo, D.H., Abdulrahman, S.A. and Kuliya-Gwarzo, A. (2016) Evaluating Laboratory Request Forms Submitted to Haematology and Blood Transfusion Departments at a Hospital in Northwest Nigeria. African Journal of Laboratory Medicine, 5, 6. https://doi.org/10.4102/ajlm.v5i1.381

[13] Adekoge, O.A., Idowa, A.A. and Jeje, O.A. (2011) Incomplete Laboratory Request Forms as a Contributory Factor to Preanalytical Errors in a Nigerian Teaching Hospital. African Journal of Biochemistry Research, 5, 82-85.

[14] Zini, G. (2014) Stability of Complete Blood Count Parameters with Storage: Toward Defined Specifications for Different Diagnostic Applications. International Journal of Laboratory Hematology, 36, 111-113. https://doi.org/10.1111/ijlh.12181

[15] Antwi-Baffour, S., Quao, E., Kyeremeh, R. and Mahmood, A.S. (2103) Prolong Storage of Blood in EDTA Has an Effect on the Morphology and Osmotic Fragility of Erythrocytes. International Journal of Biomedical Science and Engineering, 1, 20-23. https://doi.org/10.11648/j.ijbse.20130102.11

[16] Nutt, L., Zemlin, A.E. and Erasmus, R.T. (2008) Incomplete Laboratory Request Forms: The Extent and Impact on Critical Results at a Tertiary Hospital in South Africa. Annals of Clinical Biochemistry, 45, 463-466. https://doi.org/10.1258/acb.2008.007252

[17] Burton, J.L. and Stephenson, T.J. (2001) Are Clinicians Failing to Supply Adequate Information When Requesting a Histopathological Investigation? Journal of Clinical Pathology, 54, 806-808. https://doi.org/10.1136/jcp.54.10.806

[18] Zemlin, A.E., Nutt, L., Burgess, L. and Erasmus, R.T. (2009) Potential for Medical Error: Incorrectly Completed Request Forms for Thyroid Function Tests Limit Pathologists' Advice to Clinicians. South African Medical Journal, 99, 668-671. https://www.ncbi.nlm.nih.gov/pubmed/200 